\title{
Providing acoustic comfort of built-up zones and rooms in urban planning
}

\author{
Vadim Bespalov ${ }^{1}$, Natalia Samarskaya ${ }^{1, *}$, Ekaterina Lysova ${ }^{1}$, and Oksana Akay ${ }^{1}$ \\ ${ }^{1}$ Architectural and Construction Academy Don State Technical University, 344022, \\ Sotsialisticheskaya str. 162, Rostov-on-Don, Russia
}

\begin{abstract}
The article discusses topical issues of acoustic comfort ensuring on residential areas in urban planning. The main problems in this area are identified, as well asa new approach to their solution is proposed. The physical and energy approach, proposed by the authors in the article, had been previously used in the field of air dedusting. In the authors' opinionusing the physical-energy approach to solve problems of providing acoustic comfort, it is possible to ensure environmental safety in urban planning. The paper presents the results of the analysis of the main sources of acoustic discomfort in the urban environment, the physical parameters of sound waves; the physical essence of the acoustic pollution process is investigated, and the stages of formation, emission, and propagation of sound waves are identified. The authors propose a mathematical dependence that expresses the probability of realizing the process of acoustic impact on the surrounding urban environment. Also, the main directions of further research are presented.
\end{abstract}

\section{Introduction}

Currently the tasks of urban planning are implemented in large-scale urban development projects, which are characterized by the tendency to form a new environmentally friendly urban environment. The management of projects for the construction of new residential complexes, the creation of urban infrastructure and modern public spaces should be based on the principles of creating comfortable living conditions for the population [1,2]. At the same time the notion of comfort includes not only the factors of transport accessibility, the presence of organized public spaces, the layout of the street network, the availability of necessary services, but also, first of all, in our opinion, environmental factors (air pollutants and physical fields exposure). Contaminants include material substances, which depending on the aggregate state, are divided into solid, liquid and gaseous. If the concentration in the atmospheric air of the urban environment exceeds the norm, such substances can have a toxic, allergic, carcinogenic, mutagenic effect on a person. Even more dangerous impact on the human body can have physical fields (acoustic, vibration, electromagnetic, radioactive) [3]. Their danger lies in the fact that, having a physical nature, the result of exposure

\footnotetext{
*Corresponding author: nat-samars@yandex.ru
} 
manifests itself at the chemical level in cells and leads to functional changes in various systems of the organism $[4,5]$.

Taking into consideration the fact that noise is a random mix of sounds of varying intensity and frequency, it should be viewed as a physical field that has a negative impact on the population. First of all this is due to the over-standard acoustic impact of various sources of the urban environment, common for the territories of populated areas [6-8]. Elevated levels of sound pressure represent a serious danger to human health. Hygienic studies have established that noise interferes with normal rest and labor activity of people and may be the cause of many diseases [9].

Designing activities that reduce the acoustic impact on the territory of populated areas is undoubtedly a laborious task. At the heart of such a task liesfirstly the choice of environmentally-efficient and energy-efficient methods, techniques and technical means of noise reduction. Studies in this field have shown that there are many effective ways to reduce acoustic impact in urban environment [10,11]. At the same time environmental efficiency as a selection criterion can acquire the same values for a whole range of engineering solutions. This indicates the inadequacy of using the optimization criterion as the only indicator of environmental efficiency. In this connection our research goal was to search for a scientific approach that would permit to consider the processes of acoustic impact from a physical point of view, which will later allow us to make a scientifically based choice of noise protection measures with the necessary set of optimization criteria.

\section{Materials and methods}

The research methods were based on the analytical generalization of known scientific and technical results. The study of the parameters of the sound waveproperties, its behavior under the influence of various factors, and also the study of the problems of acoustic impact reducing and technical means improving are reflected in the works of Aistov V.A., Alekseev S.P., Anderson J., Arenas J., Berfina G.P., Bolkhovitina M.M., Brookmaer F., Vasiliev A.V., Gorodkov A.V., Gusev V.P., Jonasson X., Egorova O.O., Zaek Z., Ivanov N. I., Kavan T., Kovrigin S.D., Kryshov S.I., Kroker M., Kurtse U., Leushin P.I., Maekava D., Menge S., Meister M., Nikolov N.D. , Osin V.A., Pospelov P.I., Prohoda A.S., Prudkov B.G., Prudnikov B.G., Rasmusen K., Redferi S., Retinger M., Samolyuk E.P., Tyurina N.V., FeoktistovO.G., Fuivara K., Tsygankov V.V., Shapshnev K.N., Shevchenko D. P., Shelkovnikov D.Yu., Shishelov T.I., Shishkin I.A., Yudin E.Ya., Yamamoto K. and many other authors. Most of these authors' studies are devoted to the assessment of the acoustic impact of transport (automobile, water, rail, aviation) as well as its reduction to the environment $[6,9,12-16]$. As the results of the research the authors propose:

- methods for calculating and designing various designs of noise protection screens and developing methods for assessing their acoustic efficiency, which represents the degree of sound pressure level reduction due to noise protection measures with respect to the initial value. At the same time it should be noted that the concept of "acoustic efficiency" has not yet found wide application in the theory and practice of providing acoustic comfort, and the concept of "sound pressure level reduction", defined as the difference between the actual and required (normative) levels at the point of urban spaceis widely used;

- study of noise protection properties of vegetation and use of green plantations as an acoustic obstacle.

To consider the processes of acoustic impact on the surrounding urban environmentfrom the physical point of view we have chosen the physical and energy concept of air dedusting as an analogue [17]. The essence of this concept is to describe the dust aerosol behavior from the position of disperse systems at the stages of its formation, isolation and distribution in the environment. The given approach allows to simulate the 
physical processes that take place with a dust aerosol and to describe them mathematically by probabilistic-statistical methods. Thus, by analogy with a dust aerosol, the sound wave is considered as the main object of investigation. According to the physical-energy concept a sound wave interacts with physical objects at the stages of its formation, radiation and propagation like a dust aerosol. Unlike dust aerosol a sound wave can not be considered from the perspective of the theory of disperse systems, however, in the process of its interaction with physical objectsit changes the values of its parameters (frequency of oscillations, propagation velocity, sound intensity). Consequently the probability of the particular stage of the acoustic impact implementation or its reduction in the urban environment can be expressed by an appropriate mathematical dependence.

\section{Results}

The physicochemical approach used by the authors to study the process of ensuring the acoustic comfort of residential areas is to consider the physical characteristics of two interrelated processes: acoustic pollution of the urban environment and its reduction [18]. Each of the processes includes several stages, depending on the characteristics of the source of acoustic impact. The main sources of acoustic impact for most major cities are - motor transport, rail and air transport, industrial enterprises (Fig. 1).

Thus if we consider the effect of any source of acoustic impact on the surrounding urban environment as a physical process, then in this process we can identify the successive stages, in each of which the sound waves change the values of their parameters. One of the main parameters of a sound wave is the intensity. The intensity of sound, expressed in terms of the amplitude of the sound pressure $p_{0}$ and the vibrational velocity $v$, is:

$$
I=\frac{p_{0} v}{2}=\frac{v^{2} \mathrm{Z}_{s}}{2}=\frac{p_{0}^{2}}{2 \mathrm{Z}_{s}}, \mathrm{~W} / \mathrm{m}^{2},
$$

where: $Z_{S}$ is the specific acoustic resistance of the medium, $\mathrm{Pa} \cdot \mathrm{s} / \mathrm{m}$.

Specific acoustic resistance $Z_{S}$ is calculated with the formula:

$$
Z_{s}=\frac{p_{s}}{u}=r \cdot c, \mathrm{~Pa} \cdot \mathrm{s} / \mathrm{m}
$$

where: $c$ is the soundspeed, $\mathrm{m} ; r$ is the mediumdensity, $\mathrm{kg} / \mathrm{m}^{3} ; p_{s}$ - sound pressure, $\mathrm{dB} ; u$ is the vibrational velocity of the medium particles, $\mathrm{m} / \mathrm{s}$.

The sound pressure $(\mathrm{dB})$ is used to measure the absolute values of the sound intensity emanating from the noise source. Sound power level $L_{w}$ is the energy transferred by a sound wave through the surface in question per unit time:

$$
L_{w}=10 \lg \left(\frac{w}{w_{0}}\right), \mathrm{dB} .
$$

where: $w$ - sound waves power near the noise source, $\mathrm{W} ; w_{0}$ is the zero value of the power of sound waves, $w_{0}=11 \mathrm{~W}$. 


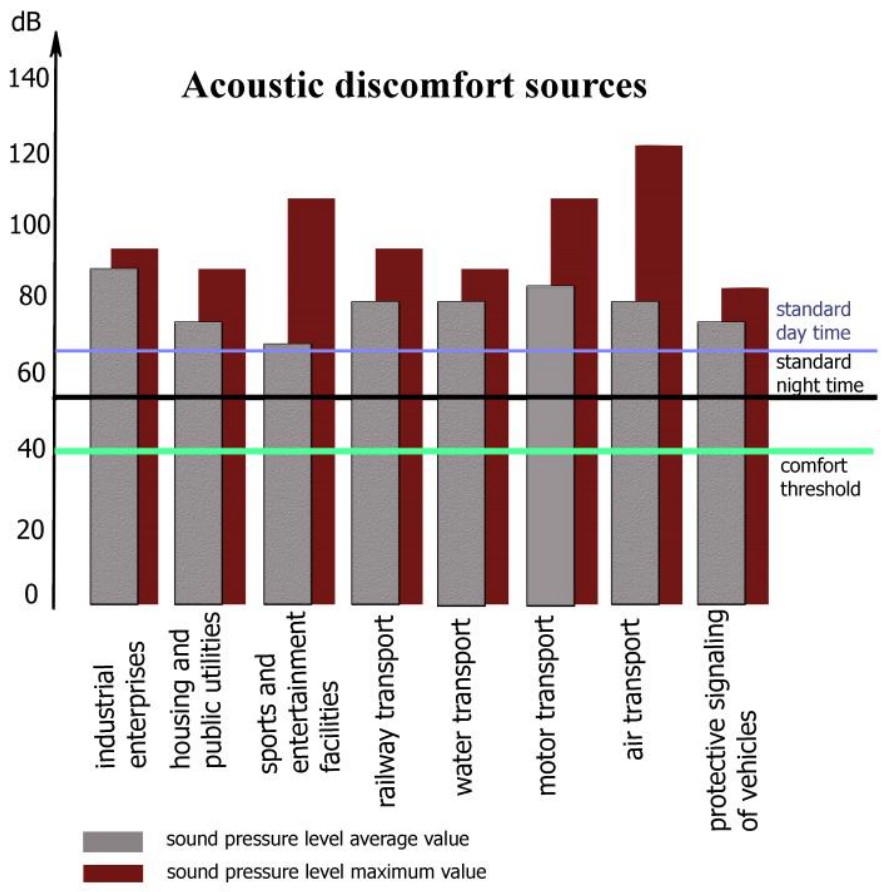

Fig. 1. The main sources of acoustic discomfort in the urban environment

Sincesound propagates in the form of a divergent spherical wave, with the passage of time and with increasing distance, the vibrations of the particles of the medium weaken, and the sound dissipates. It is also important to consider that diffraction, that is, distortion of sound rays, has a large influence on the sound propagationrange. The more diverse the medium is, the more the sound ray bends and, consequently, the shorter the range of sound propagation becomes. The speed of propagation of sound waves in a medium characterizes thesound speed, which as a ruleis less in gases than in liquids and in liquids the sound speed is less than in solids.

The sound speed in any medium is calculated with the formula:

$$
c=\sqrt{\frac{1}{\beta \rho}}, \mathrm{m} / \mathrm{s}
$$

where: $\beta$ is adiabatic compressibility of the medium, $\mathrm{Pa}-1 ; \rho$ is the density of the medium, $\mathrm{kg} / \mathrm{m}^{3}$.

Thus from the moment of formation and emission of a sound wave to its complete dispersion, the sound wave interacts with physical objects. As a result, the sound wave changes its parameters (oscillation frequency, propagation velocity, sound intensity). Therefore the process of acoustic influence of a noise source on a built-up area can be considered in stages. We have identified five main stages of the process: formation, internal radiation, internal propagation, external radiation, and external propagation of sound waves.

At the first stage the source of thesound waves formation begins to perform the oscillatory process, setting the initial parameters characterizing the sound wave 1 . The source of the sound waves formation can be a part of the technological equipment, the functional element of the vehicle engine, etc., that is, the place where the oscillatory process begins. In this case the zone of the sound waves formation can be point, linear or planar. 
At the second stage sound waves are emitted, as a rule, into the internal air medium (the carhood, the internal volume of the running gear of the rolling train, the air in the production room, etc.). When vibrating the source of the sound waves formationcauses oscillations of adjacent particles of the medium with the same frequency, which determines the second stage - the "internal" radiation of the sound wave. The state of the oscillatory motion is successively transmitted to increasingly more distant particles from the source, that is, a sound wave 2 is emitted with a frequency of oscillations equal to the frequency of its source and with a velocity determined by the properties of the air medium (molar mass and heat capacity, thermodynamic temperature). The internal source of radiation will be the zone of technological equipment, which is directly connected with the air of the working area. At the third stage the spatial distribution of sound waves in the internal volume (room, engine compartment of the car) occurs. At the stage of "internal" propagation, a number of factors affect the parameters of sound wave 3: the humidity and air mobility, the molecular structure of the gaseous medium, the phenomena of refraction and sound reflectionat the boundary of two media. In addition, any medium has a viscosity, therefore sound attenuation is observed, i. e. the decrease in its amplitude, and consequently the intensity of the sound waves as they propagate.

At the fourth stage the sound waves are emitted into the air of the surface layer of the atmosphere outside the interior volume of the room, the engine compartment of the car, etc. The zone of the production room (the carhood), which is directly connected with the air of the surface layer of the atmosphere and through which the sound waves emit into the external environment, is an external source of radiation.

At the fourth stage of "external" radiation of sound waves in the air of the surface layer of the atmosphere, the parameters of the sound wave 4 under the influence of physical objects participating in the process of acoustic pollution of the urban environment are analogous to the second stage.

At the fifth stage, the spatial distribution of sound waves in the air of the surface layer of the atmosphere outside the premises or vehicle occurs. At the same time at the fifth stage the number of factors influencing the parameters of sound waves increases significantly. The process of acoustic impact at this stage is due to aerodynamic properties of the external air environment, features of the terrain and urban buildings.

Thus, a step-by-step analysis of the process of acoustic impact allows us to consider the change in the physical parameters of sound waves (oscillationsfrequency, propagation velocity, soundintensity) in the process of their interaction with physical objects. In view of the wide variety of sources of acoustic impact in the urban environmentas well as the specific features of these sources the process under investigation may not include all stages. For example there may be no stages of "internal" radiation and propagation. However for each type of source (industrial equipment, automobile, rail, air transport, etc.) the number of stages in the process of acoustic impact on the urban environment will be the same.

Mathematically the physical essence of the process of acoustic impact on the residential area of the urban environment, taking into account the possibility of implementing the corresponding dependent successive events, can be expressed by the following formula:

$$
P_{\text {polA }}=P_{\text {formA }} \cdot P_{\text {radA }(\text { complA })} \cdot P_{\text {propA(radA })}
$$

where $P_{\text {pola }}$ is the probability of the surrounding urban environmentacoustic pollution process; $P_{\text {formA }}$ is the probability of sound wavesformation stage; $P_{\text {radA(complA) }}$ is the probability of sound wavesradiation stage, provided that the stage of formation is completed; $P_{\text {propA(radA) }}$ is the probability of sound waves spatial propagation stage the under the condition that the radiation stage is completed. 


\section{Discussion}

The physical and energy approachapplied by the authors to study the process of acoustic influence on the surrounding urban environment of various sources allows us to analyze this process step by step, revealing changes in the parameters of sound waves at each stage. Knowing the parameters of sound waves and the characteristics of the physical objects interacting with them, one can purposefully exert an external suppressive influence on sound waves at each stage and achieve a decrease in the acoustic pollution of the urban environment.

The main contribution to the development of this field of science, according to the authors, is the application of a scientifically sound approach to solving the problems of providing acoustic comfort of built-up areas, especially at the stage of urban planning [19]. Further development of the physical and energy approach based on the systematization of the main sources of acoustic pollution, physical parameters of sound waves and consideration of the physical nature of the process will allow to conduct modeling the physical process of acoustic impact for any conditions of the urban environment. And later on, based on the models obtained, to develop physical models for reducing acoustic impact, as well as scientifically justify the criteria for assessing and selecting the most environmentally efficient and energy-efficient means and ways to reduce city noise.

\section{Conclusions}

Providing the acoustic comfort ofbuilt-up areas in urban planning is a very urgent task [20]. Scientific researches to combat noise in the urban environment have been carried out everywhere throughout the world, mainly, in the direction of assessing the acoustic impact of various modes of transport and reducing it, including studies of acoustic obstructions various types physical characteristics and development of methods for assessing their acoustic efficiency. At the same time there is no single list of criteria and methods for choosing noise protection measures. Due to thiswe proposed to use the physical-energy approach to solve the problems of providing acoustic comfort in the urban environment.

The physical and energy approach allows to simulate physical processes that occur with sound waves at the stages of formation, radiation and propagation and also mathematically describe them by probabilistic-statistical methods. For the first time such an approach is suggested by the authors for application in order to provide acoustic comfort in urban environment. Previously the physical and energy approach had been used to solve the problems of reducing air pollution by solid, liquid and gaseous substances.

Thus at this stage of research the following goals have been reached by the authors:

- the main sources of acoustic pollution in the urban environment have beensystematized;

- the physical parameters of sound waves have been studied;

- the physical essence of the process of acoustic pollution has been studied, as well as the stages of formation, emission, and propagation of sound waves have been singled out; - the mathematical dependence expressing the probability of acoustic influence realization process on the surrounding urban environment has been proposed.

Based on the results obtained by the authors, it is planned to solve the following problems in the future:

- to develop physical models of acoustic pollution and its reduction for different types of sources in the urban environment;

- to develop a classification scheme for the system to reduce the acoustic pollution of the urban environment and the evaluation criteria that determine the degree of ecological safety 
of the environment of built-up areas by the noise factor at each stage of the noise reductionprocess;

- to create a scientifically grounded methodology for choosing the optimal system for reducing acoustic pollution based on the methodology for estimating, calculating and designing environmentally efficient and energy-efficient systems for providing acoustic comfort in the urban environment.

The solution of the set tasks will allow to provide acoustic comfort of built-up areas in urban planning on the basis of the creation of a scientific concept and a methodological base for calculating and designing environmentally efficient and energy-efficient noise reduction technologies.

\section{References}

1. S. A. Lebedeva and others. Inženernyj vestnik Dona (Rus), 2-1 URL: ivdon.ru/en/magazine/archive/n2y2015/2894 (2015)

2. T. V. Poljakova, A. V. Sajbel, S. V. Halezin. Inženernyj vestnik Dona (Rus), 4-2 URL: ivdon.ru/magazine/archive/n4p2y2012/1388 (2012)

3. V. I. Bespalov, I. V. Sherbet. Scientific Review, 9(3), 722-726 (2014)

4. S. Luzzi, A. V. Vasiliev. Ecology and Industry of Russia, 3, 58-63 (2016)

5. G. Brambilla, D. Casini, S. Secchi, G. Cellai, 24th International Congress on Sound and Vibration, ICSV (2017).

6. N. I. Ivanov, D. A. Kuklin, P.V. Matveyev. Ecology and life safety of industrialtransport complexes, 7, 179-184 (2015).

7. E. N. Belaja. Modern Science: Modern State and Ways of Development, 8, 130-134 (2016)

8. V. A. Alyabyeva, E.A. Losminskaya, S. A. Grin. Young, 51 (11), (2017)

9. V. V. Bulking, I. N. Kirillov, T. D. Shchelokov. Methods and devices of information transmission and processing, 18, 22-26, (2016)

10. D. Casini, G. Cellai, S. Secchi, J. Fogola, F. Scamoni. Paper presented at the 22nd International Congress on Sound and Vibration, ICSV (2015)

11. V. B. Bulkin, M. V. Kalinichenko, I. N. Salnikov. Noise Theory and Practice, 1 (1), 3241 (2015)

12. N. I. Ivanov, A. E. Shashurin, Yu. S. Boyko. Noise Theory and Practice, V. 2, 4 (6), 24-28 (2016)

13. S. Secchi, F. Asdrubali, G. Cellai, A. Rotili, I. Vannucchi. Journal of Building Engineering 5, 1-12 (2016)

14. P. H. Trombetta Zannin. Noise Pollution in Urban and Industrial Environments: Measurements and Noise Mapping, 1-342 (2016)

15. N. I. Ivanov, A. E. Shashurin, Yu. S. Boyko. In the proceedings: Protection against increased noise and vibration, a collection of reports. Baltic State Technical University "Voenmeh", 129-135 (2017)

16. P. E. Kirrian Fiedler, P. H. Trombetta Zannin. Noise Pollution in Urban and Industrial Environments: Measurements and Noise Mapping, 33-50 (2016)

17. V. I. Bespalov, N. S. Samarskaya, O. S. Gurova. Proceedings of the International Scientific and Practical Conference "Modern Directions of Theoretical and Applied Research ", March 18-30, 1 (3), 10-15 (2014) 
18. V. I. Bespalov, N. S. Samarskaya, E. P. Lysova. Inženernyj vestnik Dona (Rus), 3, URL: http://www.ivdon.ru/en/magazine/archive/n3y2015/3219 (2015)

19. V. I. Bespalov, N. S. Samarskaya, G.A. Visnevsky. Inženernyj vestnik Dona (Rus), 4, URL: http://www.ivdon.ru/en/magazine/archive/n4y2017/4395 (2017)

20. P. H. Trombetta Zannin, F. Bunn. Journal of Environmental Health Science and Engineering 12 (1), 14 (2014). 